\title{
EFEITO DA TEMPERATURA NA REAÇÃO DE DESIDRATAÇÃO DA FRUTOSE CATALISADA POR ÁCIDOS DE LEWIS E BRØNSTED
}

\author{
J. B. dos Santos ${ }^{1 *}$, D. P. Barbosa ${ }^{1}$, M. R. Meneghetti ${ }^{1}$ e S. M. P. Meneghetti ${ }^{1}$ \\ ${ }^{1}$ Universidade Federal de Alagoas, Instituto de Química e Biotecnologia \\ E-mail para contato: jailmabs@ @otmail.com
}

\begin{abstract}
RESUMO - Atingir objetivos de sustentabilidade exigirá mudanças significativas no fornecimento de um conjunto de insumos visando à busca por tecnologias verdes. A biomassa é um excelente candidato para produção de combustíveis e insumos químicos devido ao potencial renovável e disponibilidade. Este trabalho explorou a atividade de diferentes catalisadores ácidos de Lewis a base de $\operatorname{Sn}(\mathrm{IV})$ e ácido sulfúrico (ácido de Brønsted) para fins comparativos, frente a reações de degradação da frutose em produtos químicos, nos tempos de reação de 30, 60, e 120 min. e temperaturas de $150{ }^{\circ} \mathrm{C}$ e $190{ }^{\circ} \mathrm{C}$. Nas duas temperaturas a conversão para os ácidos de Lewis foi maior do que o ácido de Brønsted, sendo que, a $190{ }^{\circ} \mathrm{C}$ em $2 \mathrm{~h}$ a conversão foi total para todos os catalisadores. A avaliação da temperatura foi importante no que se refere a conversão e rendimentos da frutose. Em todas as temperaturas, o perfil de produtos formados foi diferente para ambos os sistemas: enquanto os ácidos de Lewis foram mais seletivos a ácido lático, o de Brønsted foi seletivo a HMF.
\end{abstract}

\section{INTRODUÇÃO}

A dificuldade de exploração das reservas de combustíveis fósseis associada à diminuição de sua disponibilidade e a crescente atenção sobre os problemas ambientais são fortes indicativos para buscar o desenvolvimento de fontes de energia sustentáveis e produtos que atendam a demanda da química fina. Neste contexto, a biomassa apresenta-se como alternativa promissora para o fornecimento sustentável de biocombustíveis e valiosos produtos químicos. De maneira especial, os carboidratos derivados da biomassa, são abundantes e promissores fontes de energia, além de relativamente baratos e renováveis. Podem ser aplicados na síntese de uma ampla gama de produtos químicos da plataforma da biorrefinaria (JADHAV et al., 2013).

O 5-hidrometilfurfural (HMF) derivado de carboidratos é um intermediário versátil e fundamental, que está atraindo muita atenção na química dos biocombustíveis e na indústria do petróleo (ZHAO et al., 2011). Além disso, os açúcares, tais como frutose, sacarose e glicose têm demonstrado constituir matérias-primas preferenciais para a produção de HMF com elevado rendimento, em presença de ácido (JADHAV et al., 2013). 


\section{9 a 22 de outubro de 2014 \\ Florianópolis/SC}

A hidrólise de materiais lignocelulósicos com ácido sulfúrico $\left(\mathrm{H}_{2} \mathrm{SO}_{4}\right)$ requer reatores resistentes à corrosão e tem problemas de eliminação de resíduos (SHIMIZU et al., 2009). Vários estudos têm sido desenvolvidos com sistemas catalíticos homogêneos e heterogêneos, tais como ácidos minerais (ASGHARI e YOSHIDA, 2006; ASGHARI e YOSHIDA, 2007), ácidos sólidos (QI et al., 2009; ZHAO et al., 2011; FAN et al., 2011; QU et al., 2012), que facilitam a desidratação da frutose com moderado a bom rendimento em HMF. No entanto, poucos estudos têm-se centrado sobre os efeitos da acidez de Brønsted e Lewis sobre a atividade catalítica e seletividade para essa reação. As relações entre a acidez de Brønsted e de Lewis e eficiência catalítica em termos de atividade e seletividade são discutidos neste trabalho. Portanto, este trabalho explorou o efeito da temperatura na atividade de diferentes catalisadores ácidos de Lewis a base de $\mathrm{Sn}$ (IV) e ácido sulfúrico (ácido de Brønsted) para fins comparativos, frente a reações de degradação da frutose em produtos químicos.

\section{SEÇÃO EXPERIMENTAL}

No âmbito desse estudo investigou-se o processo de hidrólise e degradação da frutose, na presença dos catalisadores ácido sulfúrico $\left(\mathrm{H}_{2} \mathrm{SO}_{4}\right)$, óxido de dibutil estanho (IV) - $\left(\mathrm{C}_{4} \mathrm{H}_{9}\right)_{2} \mathrm{SnO}-$ (DBTO) e Dibutil dilaurato de estanho (IV) - $\left(\mathrm{C}_{4} \mathrm{H}_{9}\right)_{2} \mathrm{SnO}\left(\mathrm{C}_{12} \mathrm{H}_{23} \mathrm{O}_{2}\right)_{2}-($ DBTDL). Os compostos foram obtidos da Sigma-Aldrich e utilizados como recebidos. $\mathrm{O}_{2} \mathrm{SO}_{4}$ foi adquirido comercialmente do Merck (grau analítico) e a D-Frutose com 100\% de pureza obtida da Vetec.

Os ensaios catalíticos foram realizados em batelada, num reator de aço inoxidável com capacidade de $100 \mathrm{~mL}$ acoplado a um manômetro, um termopar para controle da temperatura e um agitador magnético trabalhando a $1000 \mathrm{rpm}$. No reator foi colocada uma solução de frutose $(0,48 \mathrm{~g}$ de frutose em $60 \mathrm{~mL}$ de água deionizada) e catalisador $\left(2,69 \times 10^{-5} \mathrm{~mol}\right)$. O sistema foi aquecido nas temperaturas de $150{ }^{\circ} \mathrm{C}$ e $190{ }^{\circ} \mathrm{C}$ e as reações foram realizadas em diferentes tempos reacionais (30, 60 e 120 min.).

Para detecção e quantificação dos diferentes produtos formados, a fase líquida foi filtrada através de filtros de 0,45 $\mu \mathrm{m}$ (MilliporeTM) e em seguida, foi analisada utilizando um sistema de HPLC ProStar 210 (Varian), equipado com coluna MetaCarb 87H (300 mm x 7,8 mm), na temperatura de $55^{\circ} \mathrm{C}$, utilizando água acidificada $\left(0,005 \mathrm{~mol} . \mathrm{L}^{-1}\right)$ com fluxo de $0,70 \mathrm{~mL} \cdot \mathrm{min} .{ }^{-1}$.

A conversão e o rendimento da frutose foram calculados de acordo com Santos et al. (2013).

\section{RESULTADOS E DISCUSSÃO}

\subsection{Conversões da Frutose}

Na Figura 1 estão apresentados os resultados obtidos para a conversão da frutose, na presença dos diversos tipos de catalisadores, para as reações conduzidas a $150{ }^{\circ} \mathrm{C}$ realizadas em três tempos reacionais $(30,60$ e $120 \mathrm{~min}$.). 


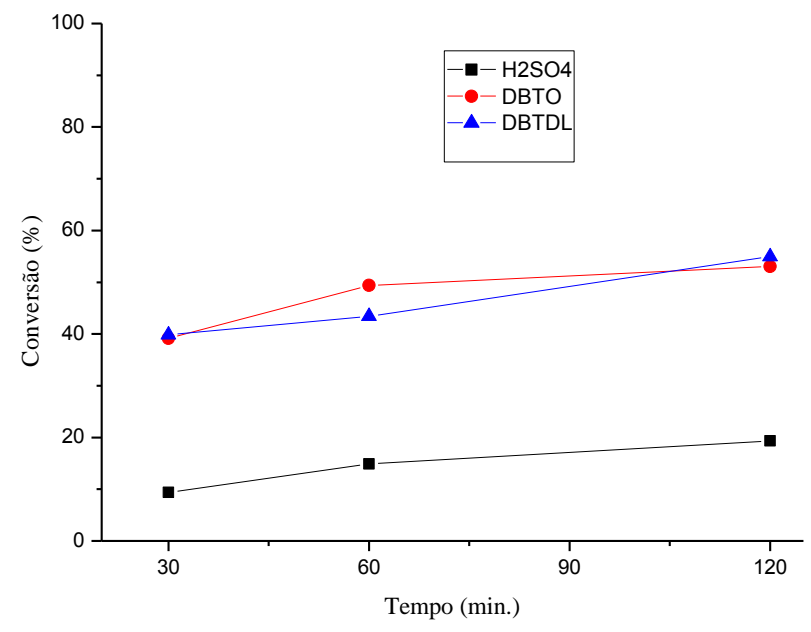

Figura 1 - Conversão da frutose para os diversos catalisadores na temperatura de $150^{\circ} \mathrm{C}$.

Na temperatura de $150{ }^{\circ} \mathrm{C}$ (Figura 1), a conversão obtida nas reações realizadas com o catalisador ácido sulfúrico aumentou à medida que o tempo reacional cresceu, mas não ultrapassou conversões maiores que $20 \%$. Para os catalisadores DBTO e DBTDL as conversões são comparáveis entre si, e variaram entre $40 \%$ e $55 \%$, aumentando de acordo com o aumento do tempo reacional para os dois sistemas catalíticos.

Na Figura 2 estão apresentados os resultados obtidos para a conversão da frutose, na presença dos diversos tipos de catalisadores, para as reações conduzidas a $190{ }^{\circ} \mathrm{C}$ realizadas nos três tempos reacionais.

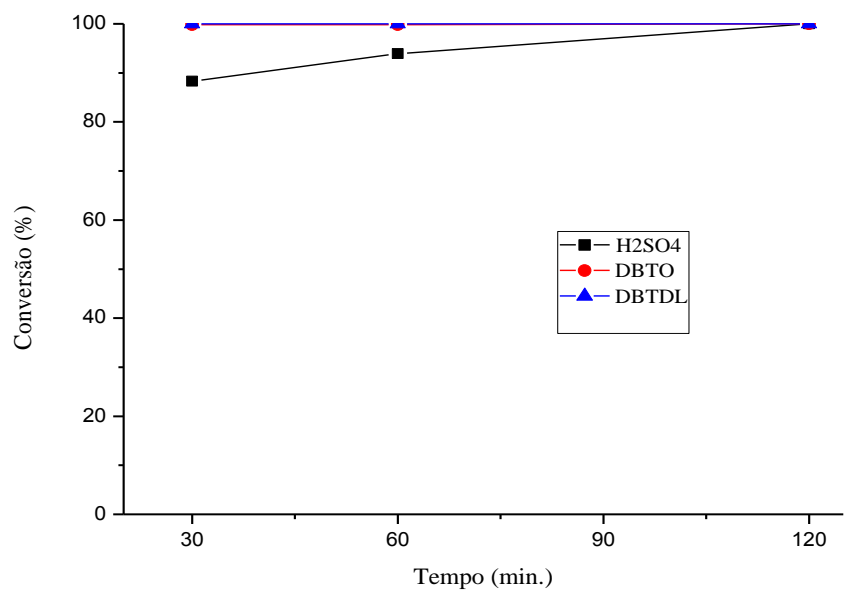

Figura 2 - Conversão da frutose para os diversos catalisadores na temperatura de $190{ }^{\circ} \mathrm{C}$. 
A $190{ }^{\circ} \mathrm{C}$ (Figura 2), para o catalisador ácido sulfúrico a conversão foi de $88 \%$ (30 min.), 94 $\%$ (60 min.) e $100 \%$ (120 min.). Porém, para os catalisadores DBTO e DBTDL a conversão foi total em todos os tempos reacionais.

Vale salientar que na temperatura de $150{ }^{\circ} \mathrm{C}$, as conversões foram inferiores a $55 \%$, porém, a tendência em termos de comportamento catalítico de todos os sistemas foi a mesma nas duas temperaturas $\left(150\right.$ e $\left.190^{\circ} \mathrm{C}\right)$, mantendo as maiores conversões para os catalisadores ácidos de Lewis.

\subsection{Produtos Formados no Processo de Degradação da Frutose}

Os produtos detectados, a partir da degradação da frutose para estas condições reacionais foram: ácido acético, ácido fórmico, ácido lático, gliceraldeido, piruvaldeido, 1,3-dihidroxiacetona e 5hidroximetilfurfural (HMF), cujos dados estão representados nas Tabelas 1 e 2 . A exceção foi a 1,3dihidroxiacetona que não foi quantificada, e, além destes, traços de galactose, 1,6 anidroglicose, ácido levulínico e furfural foram detectados em condições variadas de reações, como por exemplo, furfural foi formado em reações de 60 e 120 min., a $190{ }^{\circ} \mathrm{C}$, quando o catalisador foi o ácido sulfúrico. Para as reações realizadas na presença dos demais catalisadores não houve a formação deste produto.

Na Tabela 1 estão apresentados os dados da frutose para os catalisadores ácidos de Brønsted e Lewis em rendimento dos produtos, na temperatura de $150^{\circ} \mathrm{C}$.

Tabela 1 - Rendimento em produtos da frutose a $150^{\circ} \mathrm{C}$

\begin{tabular}{|c|c|c|c|c|c|c|c|c|}
\hline Temp & Catalis & $\begin{array}{c}\text { Tempo } \\
\text { (min) }\end{array}$ & $\begin{array}{c}\text { HMF } \\
(\%)\end{array}$ & $\begin{array}{c}\text { Ac } \\
\text { Lát } \\
(\%)\end{array}$ & $\begin{array}{c}\text { Ac } \\
\text { Fórm } \\
(\%)\end{array}$ & $\begin{array}{c}\text { Ac } \\
\text { Acét } \\
(\%)\end{array}$ & $\begin{array}{c}\text { Glicerald } \\
(\%)\end{array}$ & $\begin{array}{c}\text { Piruvald } \\
(\%)\end{array}$ \\
\hline \multirow{9}{*}{$150^{\circ} \mathrm{C}$} & \multirow{3}{*}{$\mathrm{H}_{2} \mathrm{SO}_{4}$} & 30 & 2,9 & ND & ND & ND & 0,5 & ND \\
\hline & & 60 & 3,9 & 0,2 & ND & ND & 1,2 & 1,5 \\
\hline & & 120 & 6,6 & 0,1 & ND & ND & ND & ND \\
\hline & \multirow{3}{*}{ DBTO } & 30 & 1,1 & 2,3 & 1,5 & 1,1 & 5,8 & 4,3 \\
\hline & & 60 & 1,8 & 5,2 & 2,7 & 2,5 & 2,5 & 4,3 \\
\hline & & 120 & 2,9 & 3,9 & 2,6 & 2,5 & 1,2 & 4,3 \\
\hline & \multirow{3}{*}{ DBTDL } & 30 & 0,5 & 0,8 & 0,0 & 0,8 & 7,8 & 3,6 \\
\hline & & 60 & 1,3 & 3,4 & 2,3 & 1,9 & 5,1 & 5,0 \\
\hline & & 120 & 2,8 & 6,4 & 3,8 & 3,6 & 2,5 & 5,4 \\
\hline
\end{tabular}

$\mathrm{ND}=$ não detectado

Para o catalisador ácido de Brønsted (Tabela 1) praticamente apenas traços de HMF foram 
observados, mas já é possível inferir que o rendimento aumenta à medida que o tempo reacional aumenta. $\mathrm{O}$ percentual de todos os produtos formados e detectados tiveram rendimentos menores que $10 \%$. Porém, esta temperatura define claramente o perfil dos produtos formados para estes sistemas catalíticos.

A reações realizadas com os catalisadores DBTO e DBTDL (Tabela 1) tiverem o mesmo comportamento em termos de rendimento e perfil de produtos formados. O teor de HMF, produto de desidratação da frutose (AINDA, et al., 2007), aumentou à medida que o tempo aumentou. Gliceraldeido, piruvaldeido, 1,3-dihidroxiacetona e ácido lático são produtos resultantes de reação retro-aldolíca da frutose (AINDA, et al., 2007). Neste caso, o gliceraldeido e o piruvaldeido tiveram maoires rendimentos. Porém, os teores de gliceraldeido diminuíram com o aumento do tempo, enquanto que o piruvaldeido aumentou à medida que o tempo cresceu. $\mathrm{O}$ quantidade de ácido acético, produto de degradação direta da frutose (ASGHARI e YOSHIDA, 2006), também aumentou com o aumento do tempo reacional e o quantitativo de ácido fórmico, produto de hidratação do HMF (AINDA, et al., 2007), tendeu também a aumentar com o aumento do tempo reacional.

$\mathrm{Na}$ Tabela 2 estão apresentados os resultados dos produtos em termos de rendimento para a temperatura de $190^{\circ} \mathrm{C}$.

Tabela 2 - Rendimento em produtos da frutose a $190^{\circ} \mathrm{C}$

\begin{tabular}{|c|c|c|c|c|c|c|c|c|}
\hline Temp & Catalis & $\begin{array}{l}\text { Tempo } \\
\text { (min.) }\end{array}$ & $\begin{array}{c}\text { HMF } \\
(\%)\end{array}$ & $\begin{array}{l}\text { Ac } \\
\text { Lát } \\
(\%)\end{array}$ & $\begin{array}{c}\text { Ac } \\
\text { Fórm } \\
(\%)\end{array}$ & $\begin{array}{c}\text { Ac } \\
\text { Acét } \\
(\%)\end{array}$ & $\begin{array}{c}\text { Glicerald } \\
(\%)\end{array}$ & $\begin{array}{c}\text { Piruvald } \\
(\%)\end{array}$ \\
\hline \multirow{9}{*}{$190{ }^{\circ} \mathrm{C}$} & \multirow{3}{*}{$\mathrm{H}_{2} \mathrm{SO}_{4}$} & 30 & 34,2 & 1,3 & 6,9 & 1,2 & $<1 \%$ & ND \\
\hline & & 60 & 30,1 & 1,6 & 9,5 & 1,7 & $<1 \%$ & ND \\
\hline & & 120 & 20,5 & 1,1 & 13,2 & 1,5 & $<1 \%$ & ND \\
\hline & \multirow{3}{*}{ DBTO } & 30 & 3,6 & 32,9 & 14,2 & 10,8 & $<1 \%$ & ND \\
\hline & & 60 & 3,4 & 38,8 & 10,9 & 13,1 & ND & ND \\
\hline & & 120 & 2,5 & 41,7 & 14,2 & 15,1 & ND & ND \\
\hline & \multirow{3}{*}{ DBTDL } & 30 & 4,1 & 36,3 & 13,4 & 11,6 & $<1 \%$ & $<1 \%$ \\
\hline & & 60 & 3,4 & 38,1 & 13,4 & 11,7 & ND & $<1 \%$ \\
\hline & & 120 & 2,0 & 37,7 & 12,6 & 11,6 & ND & ND \\
\hline
\end{tabular}

$\mathrm{ND}=$ não detectado

A $190{ }^{\circ} \mathrm{C}$ (Tabela 2), o rendimento de conversão da frutose quando usou o catalisador ácido foi 


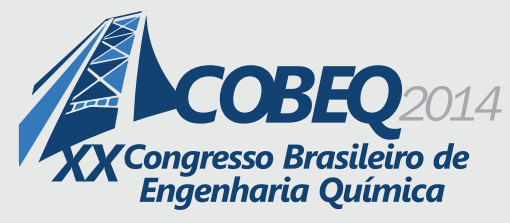

19 a 22 de outubro de 2014
Florianópolis/SC

maior em todos os tempos para o HMF, em relação aos demais produtos para este catalisador, sendo que, ao contrário da temperatura de $150{ }^{\circ} \mathrm{C}$, o rendimento diminuiu à medida que o tempo reacional aumentou. Já com o emprego dos complexos metálicos, o rendimento em HMF foi menor que $5 \%$ em todos os tempos de reação. Porém, o rendimento dos ácidos lático, fórmico e acético foi significativo em relação ao catalisador ácido sulfúrico (Tabela 2). Os dois complexos metálicos, apresentaram comportamento semelhantes em termos de percentual de produtos formados, sendo que, o catalisador DBTO parece seguir uma tendência de aumentar o rendimento à medida que o tempo aumenta, o que difere do catalisador DBTDL que aparentemente não tende a aumentar com o tempo, ou seja, nos primeiros 30 min. de reação já se atinge rendimentos semelhantes a 120 min.

Diante dos resultados expostos, é possível observar a importância da avaliação da temperatura neste estudo, tanto em termos de conversão como em termos de rendimento da frutose. No que se refere a conversão, a $150{ }^{\circ} \mathrm{C}$ (Figura 1 ) as conversões para os sistemas catalíticos foram inferiores a $60 \%$, sendo que o catalisador ácido sulfúrico a maior conversão foi $20 \%$. A $190{ }^{\circ} \mathrm{C}$ (Figura 2), todos os sistemas catalíticos obtiveram conversões de $100 \%$, com exceção do $\mathrm{H}_{2} \mathrm{SO}_{4}$ em 30 min. (88 \%) e 60 min. (94\%). Ao analisar o rendimento, em $150{ }^{\circ} \mathrm{C}$, observa-se rendimentos inferiores a $10 \%$, porém, detecta-se produtos como gliceraldeido, piruvaldeido e 1,3-dihidroxiacetona, os quais não são detectados na temperatura de $190{ }^{\circ} \mathrm{C}$. Ainda a $190{ }^{\circ} \mathrm{C}$, distingui-se claramente a tendência da atividade catalítica dos catalisadores ácidos de Lewis frente ao de Brønsted, ou seja, enquanto os ácidos de Lewis são mais seletivos a ácido lático, o ácido de Brønsted é seletivo a HMF. Outro fator importante para os ácidos de Lewis, usados neste estudo, em termos de atividade, é o emprego de condições reacionais brandas (em termos de temperatura e pressão), visto que a literatura aborda estudos onde o perfil de produtos formados foi semelhante a este trabalho, porém, necessitou-se de temperaturas superiores a $300{ }^{\circ} \mathrm{C}$ e pressões de $100 \mathrm{MPa}$, em reações de desidratação da frutose sem uso de catalisador, e além, com rendimentos inferiores (AINDA, et al., 2007).

\section{CONCLUSÕES}

Os catalisadores ácidos de Lewis, de uma maneira geral, tiveram rendimentos comparáveis entre si. Desempenharam papel importante em termos de atividade nas reações de degradação da frutose, em termos de conversão e rendimento em produtos, com maior percentual em ácido lático diferenciando da atividade do ácido de Brønsted que foi seletivo a HMF. Os complexos metálicos estudados mostram-se eficientes em reações de desidratação da frutose na diminuição das condições reacionais (temperatura e pressão) frente a reações de desidratação da frutose sem uso do catalisador quando se comprara com a literatura.

A temperatura também desempenhou papel positivo tanto para a conversão como para o rendimento da frutose. Nas duas temperaturas, o perfil de produtos formados foi diferente para ambos os sistemas, sendo que na maior temperatura atingiram-se maiores rendimentos e conversões.

\section{AGRADECIMENTOS}

\section{UFAL, IQB, CAPES, CNPq, FINEP.}




\section{REFERÊNCIAS}

AIDA, T. M.; TAJIMA, K.; WATANABE, M.; SAITO, Y.; KURODA, K.; NONAKA, T.; HATTORI, H.; SMITH JR., R. L.; ARAI, K. Reactions of d-fructose in water at temperatures up to $400{ }^{\circ} \mathrm{C}$ and pressures up to 100MPa. J. of Supercritical Fluids, 42, 110-119, 2007.

ASGHARI, F. S.; YOSHIDA, H. Acid-Catalyzed Production of 5-Hydroxymethyl Furfural from dFructose in Subcritical Water. Ind. Eng. Chem. Res., 45, 7, 2163-2173, 2006.

ASGHARI, F. S.; YOSHIDA, H. Kinetics of the Decomposition of Fructose Catalyzed by Hydrochloric Acid in Subcritical Water: Formation of 5-Hydroxymethylfurfural, Levulinic, and Formic Acids. Ind. Eng. Chem. Res., 46, 23, 7703-7710, 2007.

FAN, C.; GUAN, H.; ZHANG, H.; WANG, J.; WANG, S.; WANG, X. Conversion of fructose and glucose into 5-hydroxymethylfurfural catalyzed by a solid heteropolyacid salt. Biomass and bioenergy, 35, 2659-2665, 2011.

JADHAV, A. H.; KIM, H.; HWANG, I. T. An efficient and heterogeneous recyclable silicotungstic acid with modified acid sites as a catalyst for conversion of fructose and sucrose into 5hydroymethylfurfural in superheated water. Bioresource Technology, 132, 342-350, 2013.

QI, X.; WATANABE, M.; AIDA, T. M.; SMITH JR. R. L. Sulfated zirconia as a solid acid catalyst for the dehydration of fructose to 5-hydroxymethylfurfural. Catalysis Communications, 10, 1771$1775,2009$.

QU, Y.; HUANG, C.; ZHANG, J.; CHEN, B.; Efficient dehydration of fructose to 5hydroxymethylfurfural catalyzed by a recyclable sulfonated organic heteropolyacid salt. Bioresource Technology, 106, 170-172, 2012.

SANTOS, J. B. DOS; BARBOSA, D. P.; MENEGHETTI, M. R.; MENEGHETTI, S. M. P. DESIDRATAÇÃO DA D-FRUTOSE CATALISADA POR COMPLEXOS A BASE DE Sn (IV). In $17^{\circ}$ Congresso Brasileiro de Catálise e VII Congresso de Catálise Mercosul, 1-5, 2013.

SHIMIZU, K.; FURUKAWA, H.; KOBAYASHI, N.; ITAYA Y.; SATSUM, A. Effects of Brønsted and Lewis acidities on activity and selectivity of heteropolyacid-based catalysts for hydrolysis of cellobiose and cellulose. Green Chem., 11, 1627-1632, 2009.

ZHAO, Q.; WANG, L.; ZHAO, S.; WANG, X.; WANG S. High selective production of 5hydroymethylfurfural from fructose by a solid heteropolyacid. catalyst. Fuel, 90, 2289-2293, 2011. 